Laser Chem., 1999, Vol. 19, pp. 117-122

Reprints available directly from the publisher Photocopying permitted by license only
(C) 1999 OPA (Overseas Publishers Association) N.V.

Published by license under the Harwood Academic Publishers imprint, part of The Gordon and Breach Publishing Group.

\title{
RECENT DEVELOPMENT OF NONLINEAR SPECTROSCOPY IN LIQUIDS
}

\author{
KEISUKE TOMINAGA \\ Institute for Molecular Science, Myodaiji, Okazaki, 444 Japan
}

(Received 25 April 1997)

\begin{abstract}
Recent developments of nonlinear spectroscopy in liquids are reviewed. Especially, offresonant fifth and seventh order nonlinear techniques are discussed in light of their application to the studies of microscopic dynamics. Vibrational echo experiments and overtone vibrational dephasing spectroscopy are examples of the higher order nonlinear spectroscopy.
\end{abstract}

Keywords: Ultrafast spectroscopy; nonlinear spectroscopy; vibrational dephasing; vibrational echo experiment; overtone vibrational dephasing

\section{INTRODUCTIOON}

Vibrational spectra get broadened in liquids compared to those in gas phase. This is caused by complex interaction between the solute and solvent molecules, and consequently molecular spectra carry, in principle, various information on microscopic details in liquid such as intermolecular interaction, structures, dynamics, so on. One traditional approach to investigate microscopic details in liquids from vibrational spectra is the lineshape analysis. However, the lineshape analysis sometimes requires certain amount of fitting parameters, especially in cases of complex microscopic models, and due to a large number of the parameters, the lineshape analysis always carries some ambiguity.

In recent several years, however, new ultrafast spectroscopies have emerged to make more rigorous approach to the vibrational line- 
shapes. Raman echo, two-dimensional Raman spectroscopy, one- and two-dimensional overtone dephasing, IR photon echo, IR pump-probe, IR grating fall into this category. Those which use Raman transitions are based one higher order, fifth and seventh order, nonlinear phenomena. In this paper we review the recent development of the nonlinear spectroscopy including the higher-order technique and its application to the liquid dynamics.

\section{VIBRATIONAL ECHO EXPERIMENTS}

In liquids homogeneous and inhomogeneous contributions to vibrational spectral broadening result from different dynamics and interactions of the molecules. Rapid perturbation to the oscillator causes the homogeneous broadening, while rather slowly varying environment around the oscillator is a source of the inhomogeneous contribution. Therefore, the determination of the relative importance of the inhomogeneity is important not only from the aspects of spectroscopy but also from the aspects of microscopic liquid dynamics. However, by the spectroscopic technique based on the third order nonlinearity the influence of nuclear dynamics on the spectra is usually masked by inhomogeneous broadening, and it is very difficult to determine the relative importance of inhomogeneity by these techniques.

It is well-known that echo technique can reveal the inhomogeneous and homogeneous contributions in the dephasing process unambiguously. As has been demonstrated in the magnetic resonance (spin echo) and the electronic spectroscopy (photon echo), the echo technique makes use of temporal two-dimensionality; a carefully chosen two excitation pulses, which are temporally separated, interact with a medium, and if the inhomogeneous contribution is dominated in the dephasing process, an echo-like signal is expected to appear. Conversely, failure of the perfect rephasing is an indication that the relative importance of the inhomogeneity is minor. Raman echo and IR photon echo are echo technique on vibrational coherence. In the Raman echo vibrational coherent state is prepared by stimulated Raman scattering, and dipole transition is used in the IR photon echo. 
Consequently, Raman echo and IR photon echo are seventh and third order nonlinear phenomena.

Berg and coworkers have demonstrated the first Raman echo experiment in liquids [1]. We have also observed the Raman echo signals from liquid benzonitrile [2]. IR photon echo experiments in liquids and glasses were also reported by Fayer and coworkers [3]. By these experiments we can study inhomogeneity of the dephasing of intramolecular high-frequency modes.

Tanimura and Mukamel proposed a temporally two-dimensional spectroscopy to determine the relative importance of the inhomogeneous contribution to dephasing of low-frequency modes [4]. In this experiment vibrational modes which are accessible by the spectral width of the laser pulse are coherently excited similarly to the impulsive stimulated Raman scattering experiments. Like other vibrational echo experiments this technique requires two excitations at different times. We have performed the first experiments of the twodimensional spectroscopy in liquid $\mathrm{CS}_{2}$ with five different short pulses in a six-wave mixing configuration [5].

\section{OVERTONE VIBRATIONAL DEPHASING EXPERIMENTS}

The vibrational quantum number, $v$, is an important factor to investigate solvent effects on vibrational transition and dephasing since the quantum number dependence of the vibrational spectrum is significantly different with dephasing mechanisms. For example, the simple stochastic theory predicts that a FWHM of the vibrational band is proportional to $v^{2}$ in the case of rapid modulation limit, whereas it is proportional to $v$ in the case of the slow modulation limit. We have performed temporally one- [6] and two-dimensional [7] overtone dephasing experiments on a few high-frequency modes, which are based on the fifth order nonlinearity. Here, we mainly discuss the onedimensional overtone experiment.

Figure 1 shows the time profiles of dephasing between the $v=2$ and 0 states of the C-D stretching of $\mathrm{CDCl}_{3}\left(\omega_{10}=2250 \mathrm{~cm}^{-1}\right)$ (a) and the sym-methyl stretching vibration of $\mathrm{CD}_{3} \mathrm{I}\left(\omega_{10}=2147 \mathrm{~cm}^{-1}\right)$ (b) both together with those of the fundamentals. Possible quantum pathways to generate the overtone signal are shown in Figure 2. For these modes 

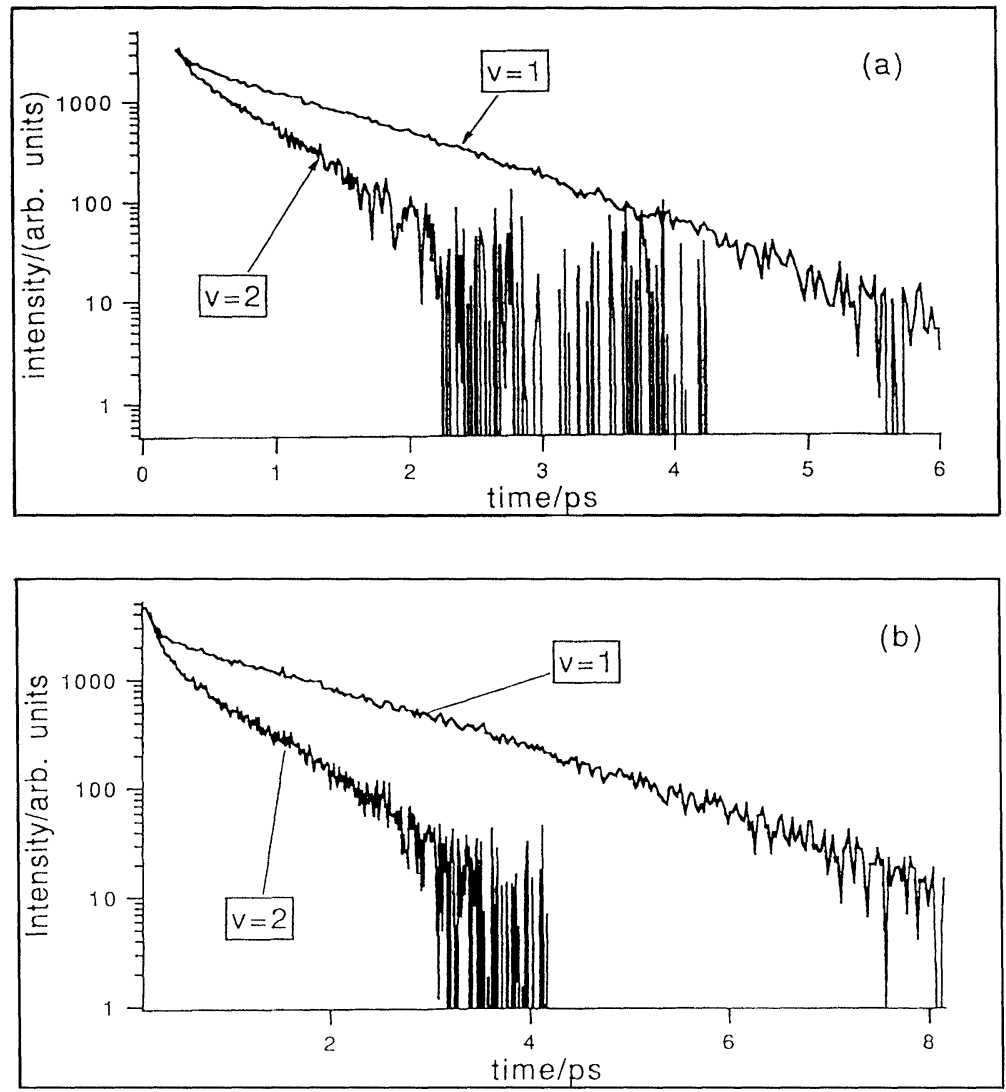

FIGURE 1 Time-profiles of the vibrational dephasing of the first overtone and fundamental bands of (a) the C-D stretching of $\mathrm{CDCl}_{3}$ and (b) the sym-methyl stretching of $\mathrm{CD}_{3} \mathrm{I}$ in neat liquids. The obtained exponential decay times are following; (a) $\tau_{v=1}=1.0 \mathrm{ps}$ and $\tau_{v=2}=0.49 \mathrm{ps}$ and (b) $\tau_{v=1}=1.6 \mathrm{ps}$ and $\tau_{v=2}=0.66 \mathrm{ps}$.

the anharmonicities $\left(\omega_{10}-\omega_{21}\right)$ are less than $10 \mathrm{~cm}^{-1}$. There are strong electronic responses in the fundamental and overtone signals around $t=0$, which are followed by nearly single exponential decays. The time constants for the single exponential decays obtained for these transients are following; for $\mathrm{CDCl}_{3} \tau_{v=1}=1.0 \mathrm{ps}$ and $\tau_{v=2}=0.49 \mathrm{ps}$, and for $\mathrm{CD}_{3} \mathrm{I} \tau_{v=1}=1.6 \mathrm{ps}$ and $\tau_{v=2}=0.66 \mathrm{ps}$.

The time profiles of both the fundamental and overtone dephasings are first analyzed in terms of a one-mode model based on the 

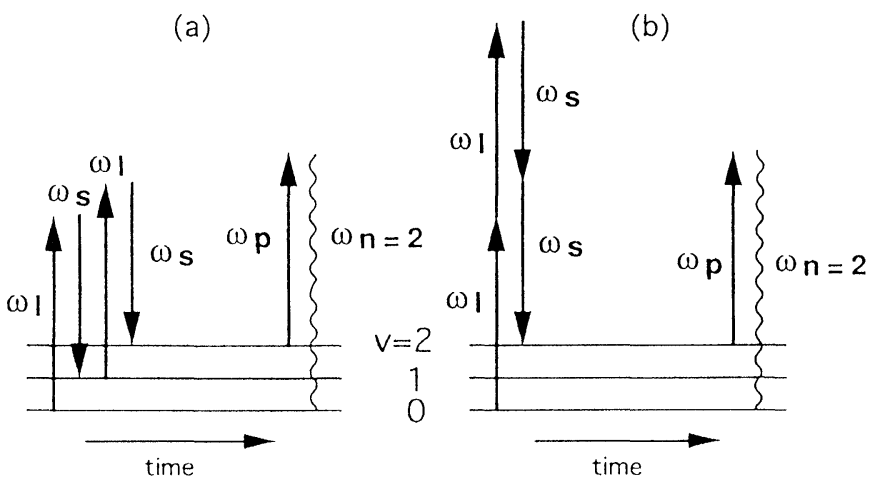

FIGURE 2 Possible quantum pathways for the overtone dephasing signal. $\omega_{l}, \omega_{s}, \omega_{p}$, $\omega_{\nu=2}$ denote the frequencies of the laser pulse, the Stokes pulse, the probe pulse, and the signal.

stochastic theory. The time correlation function of the fluctuation of the transition frequency between $v=n$ and $m$ levels is expressed,

$$
<\Delta \omega_{n m}(0) \Delta \omega_{n m}(t)>=(n-m)^{2} D^{2} \exp (-t / \tau) .
$$

The time profile of the $v$-th overtone signal is given by

$$
I(t ; v) \propto \exp \left(-2 v^{2} D^{2} \tau t\{1-t / \tau[1-\exp (-t / \tau)]\}\right)
$$

This expression predicts that a vibrational dephasing rate of the $v$-th overtone is proportional to $v^{2}$ in the rapid modulation limit, and has been used to interpret dependencies of vibrational band widths on the quantum number. Since the experimentally obtained time profiles decay single exponentially, it is natural to assume that both of the observed vibrational dynamics are in the rapid modulation limit. However, the ratio of the dephasing rates between the overtone and fundamental is 2.1 for $\mathrm{CDCl}_{3}$ and 2.4 for $\mathrm{CD}_{3} \mathrm{I}$, which is significantly smaller than four, the value expected for the rapid modulation case. As a conclusion, the simple extension of the stochastic model cannot explain the fundamental and overtone dephasings simultaneously. 


\section{References}

[1] Vanden Bout, D., Muller, L. J. and Berg, M. (1991). Phys. Rev. Lett., 67, 3700.

[2] Inaba, R., Tominaga, K., Tasumi, M., Nelson, K. A. and Yoshihara, K. (1993). Chem. Phys. Lett., 211, 183.

[3] Tokmakoff, A. and Fayer, M. D. (1995). Acc. Chem. Res., 28, 437.

[4] Tanimura, Y. and Mukamel, S. (1993). J. Chem. Phys., 99, 9496.

[5] Tominaga, K. and Yoshihara, K. (1995). Phys. Rev. Lett., 74, 3061; Tominaga, K. et al. (1995). J. Raman Spec., 26, 495; Tominaga, K. and Yoshihara, K. (1996). J. Chem. Phys., 104, 4419, ibid, 1159.

[6] Tominaga, K. and Yoshihara, K. (1996). Phys. Rev. Lett., 76, 987.

[7] Tominaga, K. and Yoshihara, K. (1997). Phys. Rev. A, 55, 831. 